\title{
SPIN WAVES TREATMENT OF THE ANTIFERROMAGNETIC GROUND STATE OF TWO ISING-LIKE SYSTEMS
}

\author{
Kunle Adegoke \\ Department of Physics, Obafemi Awolowo University, Ile-Ife, Nigeria \\ E-mail: adegoke00@gmail.com
}

(Received September 11, 2013)

\begin{abstract}
Using Anderson's spin wave theory, we derive expressions for the ground state energy of two Ising-like systems. Antiferromagnetic long range order is predicted for one of the systems.
\end{abstract}

\section{INTRODUCTION}

The analytical determination of the exact ground state of Ising-like models has proved and has remained difficult. Consequently one often has to resort to various approximate theories. One such theory is the spin waves theory introduced by BLOCH [1] in his theory of ferromagnetism and later rederived by KRAMERS and HELLER [2] in a semiclassical fashion. This theory was employed by HULTHÉN [3] in studying the small vibrations of simple antiferromagnetic lattices from their classical equilibrium state. His neglect of the zero-point energy and motion however made the results to disagree with the rigorous exact ground state worked out by BETHE [4]. The spin waves theory was used by ANDERSON [5] in successfully obtaining the ground state energy as well as in the determination of the long range order parameters of an Heisenberg antiferromagnet. The Anderson ground state energy fell within the rigorous limits derived using the variational principle and was in good agreement with the exact result obtained by Bethe for a spin-1/2 linear chain. Anderson found that the Heisenberg antiferromagnet with nearest neighbour interaction possesses no long range order in one dimension but that long range order exists in two and in three dimensions.

A more recent example of a successful application of the spin wave theory may be found in the work of GAIDIDAE and BUETTNER [6] where it was shown that the ground state properties of a frustrated compressible antiferromagnet differ qualitatively from that of an Heisenberg antiferromagnet on an anisotropic triangular lattice.

In this paper we will adapt the spin waves theory of Anderson to determine the ground state energy and other properties of the one dimensional Axial Next Nearest Neighbour Ising (ANNNI) model and the ANNNI model in a transverse field.

\section{THE ONE-DIMENSIONAL SPIN-1/2 ANNNI MODEL}

The one-dimensional spin-1/2 ANNNI model is described by the Hamiltonian 


$$
H=\sum_{i=1}^{N} S_{i}^{z} S_{i+1}^{z}+j \sum_{i=1}^{N} S_{i}^{z} S_{i+2}^{z}
$$

where $i$ and $i+1$ in the first sum refer to nearest neighbour spins and $i$ and $i+2$ in the second sum denote next nearest neighbour spins, with the summation going over all such pairs. $N$ is the number of lattice sites and $j$ is the next nearest neighbour exchange interaction. We assume periodic boundary conditions, so that $S_{N+1}^{z}=S_{1}^{z}$ and $S_{N+2}^{z}=S_{2}^{z}$.

The basic assumption in the derivation of the semiclassical spin waves is that the antiferromagnetic state is not greatly different from the classical ground state in which the spins on odd sites all point in one direction (say $+z$ ), the spins on even lattice sites in the other direction [5]. For convenience we label spins on odd sites with subscript $m$ and those on even numbered sites with $n$. So we assume

$$
S_{m}^{z} \cong+\frac{1}{2}, \quad S_{n}^{z} \cong-\frac{1}{2}
$$

$$
\begin{aligned}
& \text { Now } \\
& \left(S^{z}\right)^{2}=\left(S_{c}\right)^{2}-\left(\left(S^{x}\right)^{2}+\left(S^{y}\right)^{2}\right),
\end{aligned}
$$

where $S_{c}=\sqrt{S(S+1)}=\sqrt{3} / 2$ is the classical total spin of a spin $-1 / 2$ atom.

For the odd and even sites we have

$$
\begin{aligned}
& S_{m}^{z}=+\sqrt{S_{c}^{2}-\left(\left(S_{m}^{x}\right)^{2}+\left(S_{m}^{y}\right)^{2}\right)} \\
& =+S_{c} \sqrt{1-\frac{\left(S_{m}^{x}\right)^{2}+\left(S_{m}^{y}\right)^{2}}{S_{c}^{2}}}
\end{aligned}
$$

and

$$
\begin{aligned}
& S_{n}^{z}=-\sqrt{S_{c}^{2}-\left(\left(S_{n}^{x}\right)^{2}+\left(S_{n}^{y}\right)^{2}\right)} \\
& =-S_{c} \sqrt{1-\frac{\left(S_{n}^{x}\right)^{2}+\left(S_{n}^{y}\right)^{2}}{S_{c}^{2}}}
\end{aligned}
$$

respectively.

Under assumption (2), the binomial theorem allows us to write

$$
\begin{aligned}
& S_{m}^{z} \cong S_{c}-\left(\left(S_{m}^{x}\right)^{2}+\left(S_{m}^{y}\right)^{2}\right) / 2 S_{c}, \\
& S_{n}^{z} \cong-S_{c}+\left(\left(S_{n}^{x}\right)^{2}+\left(S_{n}^{y}\right)^{2}\right) / 2 S_{c} .
\end{aligned}
$$

For the next nearest neighbour spins we have

$$
\begin{array}{ll}
S_{i+2}^{z} \equiv S_{m}^{z} & \text { if } \mathrm{i} \text { is odd } \\
S_{i+2}^{z} \equiv S_{n}^{z} & \text { if } \mathrm{i} \text { is even. }
\end{array}
$$

Substituting (6), (7) and (8) in the Hamiltonian (1), we have

$$
H=(j-1)\left\{N S_{c}^{2}-\sum_{m}\left(\left(S_{m}^{x}\right)^{2}+\left(S_{m}^{y}\right)^{2}\right)-\sum_{n}\left(\left(S_{n}^{x}\right)^{2}+\left(S_{n}^{y}\right)^{2}\right)\right\} .
$$

Next we introduce two sets of spin waves, one pair for each sublattice: 


$$
\begin{aligned}
& S_{m}^{x}=1 / \sqrt{N} \sum_{\lambda} \exp (i \lambda m) Q_{\lambda} \\
& S_{m}^{y}=1 / \sqrt{N} \sum_{\lambda} \exp (-i \lambda m) P_{\lambda}
\end{aligned}
$$

and

$$
\begin{aligned}
& S_{n}^{x}=1 / \sqrt{N} \sum_{\lambda} \exp (-i \lambda n) R_{\lambda}, \\
& S_{n}^{y}=-1 / \sqrt{N} \sum_{\lambda} \exp (i \lambda n) S_{\lambda}
\end{aligned}
$$

where in each case the sum runs over $N / 2$ values of $\lambda$ and the wave numbers $\lambda$ are given by

$$
\lambda=2 \pi / / N, l=-N / 2,-N / 2+2, \cdots,-2,0,2, \cdots N / 2
$$

The inverse transformations are

$$
\begin{aligned}
& Q_{\lambda}=2 / \sqrt{N} \sum_{m} \exp (-i \lambda m) S_{m}^{x}, \\
& P_{\lambda}=2 / \sqrt{N} \sum_{m} \exp (i \lambda m) S_{m}^{y}
\end{aligned}
$$

and

$$
\begin{aligned}
& R_{\lambda}=2 / \sqrt{N} \sum_{n} \exp (i \lambda n) S_{n}^{x}, \\
& S_{\lambda}=-2 / \sqrt{N} \sum_{n} \exp (-i \lambda n) S_{n}^{y}
\end{aligned}
$$

Clearly, spin waves operators corresponding to spins on different sites commute, so that $Q_{\lambda}$ commutes with $Q_{\lambda^{\prime}}, P_{\lambda^{\prime}}, R_{\lambda^{\prime}}$, and $S_{\lambda^{\prime}} ; R_{\lambda}$ commutes with $R_{\lambda^{\prime}}, P_{\lambda^{\prime}}, S_{\lambda^{\prime}}$, and $Q_{\lambda^{\prime}}$ and so on.

Direct substitution of (10) and (11) into (13) and (14) together with the identity

$$
\sum_{m} \exp \left(i\left(\lambda-\lambda^{\prime}\right) m\right)=(N / 2) \delta_{\lambda \lambda^{\prime}}
$$

lead to

$$
\left[Q_{\lambda}, P_{\lambda^{\prime}}\right]=\delta_{\lambda \lambda^{\prime}} i \sum_{m} S_{m}^{z} /(N / 4)=i \delta_{\lambda \lambda^{\prime}}
$$

and

$$
\left[R_{\lambda}, S_{\lambda^{\prime}}\right]=i \delta_{\lambda \lambda^{\prime}}
$$

where we have used the assumption (2) to evaluate

$$
\sum_{m} S_{m}^{z}=\sum_{m=1}^{N / 2}\left(\frac{1}{2}\right)=\frac{N}{4} .
$$

We also have [1]

$$
\begin{aligned}
& \sum_{m}\left(S_{m}^{x}\right)^{2}=(1 / 2) \sum_{\lambda} Q_{\lambda}^{2}, \\
& \sum_{m}\left(S_{m}^{y}\right)^{2}=(1 / 2) \sum_{\lambda} P_{\lambda}^{2}, \\
& \sum_{n}\left(S_{n}^{x}\right)^{2}=(1 / 2) \sum_{\lambda} R_{\lambda}^{2},
\end{aligned}
$$

and 


$$
\sum_{n}\left(S_{n}^{y}\right)^{2}=(1 / 2) \sum_{\lambda} S_{\lambda}^{2}
$$

Substituting the spin waves (10) and (11) in (9), the sums can be easily rewritten in terms of the spin wave operators $Q_{\lambda}, P_{\lambda}, R_{\lambda}$ and $S_{\lambda}$, with the use of the set of equations (19).

The Hamiltonian then becomes

$$
H=(j-1)\left\{N S_{c}^{2}-(1 / 2) \sum_{\lambda}\left(P_{\lambda}^{2}+Q_{\lambda}^{2}+R_{\lambda}^{2}+S_{\lambda}^{2}\right)\right\} .
$$

Let us now introduce a new set of coordinates by defining [1]

$$
\begin{aligned}
& P_{\lambda}=\left(p_{1 \lambda}+p_{2 \lambda}\right) / \sqrt{2}, Q_{\lambda}=\left(q_{1 \lambda}+q_{2 \lambda}\right) / \sqrt{2}, \\
& S_{\lambda}=\left(p_{1 \lambda}-p_{2 \lambda}\right) / \sqrt{2}, R_{\lambda}=\left(q_{1 \lambda}-q_{2 \lambda}\right) / \sqrt{2} .
\end{aligned}
$$

This is a canonical transformation of the spin coordinates since $q_{1 \lambda}, p_{1 \lambda}, q_{2 \lambda}$ and $p_{2 \lambda}$ obey the same commutation rules as $Q_{\lambda}, P_{\lambda}, R_{\lambda}$ and $S_{\lambda}$

$$
\begin{aligned}
& {\left[q_{1 \lambda}, p_{1 \lambda}\right]=i,} \\
& {\left[q_{2 \lambda}, p_{2 \lambda}\right]=i \text { etc. }}
\end{aligned}
$$

The Hamiltonian is now

$$
H=(j-1)\left\{3 N / 4-(1 / 2) \sum_{\lambda}\left(q_{1 \lambda}^{2}+p_{1 \lambda}^{2}+q_{2 \lambda}^{2}+p_{2 \lambda}^{2}\right)\right\} .
$$

Since the eigenenergy of the unit-frequency harmonic oscillator $H=p_{\lambda}^{2}+q_{\lambda}^{2}$ with $\left[q_{\lambda}, p_{\lambda}\right]=\mathrm{i}$ is $E=2 n_{\lambda}+1$, the Hamiltonian (22) therefore has the energies

$$
E=(j-1)\left\{3 N / 4-\sum_{\lambda}\left(n_{1 \lambda}+n_{2 \lambda}+1\right)\right\} \text {. }
$$

In the ground state all $n_{\lambda}=0$ and we have

$$
E_{g}=(j-1) N / 4 \text {. }
$$

\section{Long range order in the one-dimensional spin-1/2 ANNNI model}

The long range order parameter is given by the expectation value of total $S_{z}$ on one site [5] in the ground state. Thus by equation (4) we have

$$
\left(S_{z}\right)_{t o t}^{(1)}=\sum_{m} S_{m}^{z}=N S_{c} / 2-\sum_{m}\left(\left(S_{m}^{x}\right)^{2}+\left(S_{m}^{y}\right)^{2}\right) / 2 S_{c} .
$$

Using (19) and the canonical transformations (21) we have

$$
\left(S_{z}\right)_{t o t}^{(1)}=1 / 2 N S_{c}-\left(1 / 8 S_{c}\right) \sum_{\lambda}\left(q_{1 \lambda}^{2}+p_{1 \lambda}^{2}+q_{2 \lambda}^{2}+p_{2 \lambda}^{2}+q_{1 \lambda} q_{2 \lambda}+p_{1 \lambda} p_{2 \lambda}\right) .
$$

The antiferromagnetic long range order parameter $\xi$ can then be computed by taking the average of $\left(S_{z}\right)_{t o t}^{(1)}$ in the ground state. Thus,

$$
\xi=1 / 2 N S_{c}-\left(1 / 8 S_{c}\right) \sum_{\lambda}\left\langle\left(q_{1 \lambda}^{2}+p_{1 \lambda}^{2}+q_{2 \lambda}^{2}+p_{2 \lambda}^{2}\right)\right\rangle
$$




$$
\begin{aligned}
& =1 / 2 N S_{c}-\left(1 / 2 S_{c}\right) \sum_{\lambda}\left\langle q_{1 \lambda}^{2}\right\rangle \\
& =1 / 2 N S_{c}-\frac{N}{8 S_{c}} .
\end{aligned}
$$

We used the fact that the average kinetic and potential energies of a harmonic oscillator are the same and that for a unit frequency oscillator, $2\left\langle q_{1 \lambda}^{2}\right\rangle=2\left\langle p_{1 \lambda}^{2}\right\rangle=1$. The magnetization per site is therefore

$$
\xi / N=12(\sqrt{3} / 2)-\frac{1}{8(\sqrt{3} / 2)}=\sqrt{3} / 6
$$

Thus we see that the spin waves theory predicts long range order for the one dimensional antiferromagnetic ANNNI model. This is in contrast for example to the Heisenberg model for which there is no long range order, as rigorously demonstrated by BETHE, HULTHÉN and later ANDERSON $[2,5,1]$.

\section{THE ONE DIMENSIONAL SPIN-1/2 ANNNI MODEL IN A STRONG TRANSVERSE FIELD}

The Hamiltonian for the transverse ANNNI model is given by

$$
H=\sum_{i} S_{i}^{z} S_{i+1}^{z}-j \sum_{i} S_{i}^{z} S_{i+2}^{z}-h_{x} \sum_{i} S_{i}^{x},
$$

where $h_{x}>0$ is the transverse external magnetic field.

Here as in the previous section we assume that the ground state of the classical model $h_{x}=0$ is antiferromagnetic. We use the binomial theorem, as in the preceding section to write

$$
S_{m}^{x} \cong S_{c}-\left(\left(S_{m}^{y}\right)^{2}+\left(S_{m}^{z}\right)^{2}\right) / 2 S_{c}
$$

and

$$
S_{n}^{x} \cong S_{c}-\left(\left(S_{n}^{y}\right)^{2}+\left(S_{n}^{z}\right)^{2}\right) / 2 S_{c} .
$$

In terms of even sites and odd sites, the Hamiltonian (29) can be written as

$$
\begin{aligned}
& H=2 \sum_{m, n} S_{m}^{z} S_{n}^{z}+j\left(\sum_{m}\left(S_{m}^{z}\right)^{2}+\sum_{n}\left(S_{n}^{z}\right)^{2}\right) \\
& -h_{x}\left(\sum_{m} S_{m}^{x}+\sum_{n} S_{n}^{x}\right) .
\end{aligned}
$$

Using (30) and (31) we have

$$
\begin{aligned}
h_{x}\left(\sum_{m} S_{m}^{x}+\sum_{n} S_{n}^{x}\right)=h_{x} N S_{c} & -\frac{h_{x}}{2 S_{c}}\left(\sum_{m}\left(S_{m}^{y}\right)^{2}+\sum_{n}\left(S_{m}^{z}\right)^{2}\right) \\
& -\frac{h_{x}}{2 S_{c}}\left(\sum_{n}\left(S_{n}^{y}\right)^{2}+\sum_{n}\left(S_{n}^{z}\right)^{2}\right) .
\end{aligned}
$$

The Hamiltonian now becomes

$$
H=-h_{x} N S_{c}+2 \sum_{m, n} S_{m}^{z} S_{n}^{z}+j\left(\sum_{m}\left(S_{m}^{z}\right)^{2}+\sum_{n}\left(S_{n}^{z}\right)^{2}\right)
$$




$$
+\frac{h_{x}}{2 S_{c}}\left(\sum_{m}\left(S_{m}^{y}\right)^{2}+\sum_{n}\left(S_{m}^{z}\right)^{2}\right)+\frac{h_{x}}{2 S_{c}}\left(\sum_{m}\left(S_{m}^{y}\right)^{2}+\sum_{n}\left(S_{m}^{z}\right)^{2}\right) .
$$

We now introduce the spin waves

$$
\begin{aligned}
& S_{m}^{y}=1 / \sqrt{N} \sum_{\lambda} \exp (-i \lambda m) Q_{\lambda}, \\
& S_{m}^{z}=1 / \sqrt{N} \sum_{\lambda} \exp (i \lambda m) P_{\lambda}, \\
& S_{n}^{y}=1 / \sqrt{N} \sum_{\lambda} \exp (i \lambda n) R_{\lambda}, \\
& S_{n}^{z}=-1 / \sqrt{N} \sum_{\lambda} \exp (-i \lambda n) S_{\lambda},
\end{aligned}
$$

with inverse

$$
\begin{aligned}
& Q_{\lambda}=2 / \sqrt{N} \sum_{m} \exp (-i \lambda m) S_{m}^{y}, \\
& P_{\lambda}=2 / \sqrt{N} \sum_{m}^{m} \exp (i \lambda m) S_{m}^{z}, \\
& R_{\lambda}=2 / \sqrt{N} \sum_{n} \exp (i \lambda n) S_{n}^{y}, \\
& S_{\lambda}=2 / \sqrt{N} \sum_{n} \exp (-i \lambda n) S_{n}^{z} .
\end{aligned}
$$

In terms of the spin waves [5],

$$
\begin{aligned}
& \sum_{m, n} S_{m}^{z} S_{n}^{z}=-\sum_{\lambda} P_{\lambda} S_{\lambda} \cos \lambda, \\
& \sum_{m}\left(S_{m}^{y}\right)^{2}=1 / 2 \sum_{\lambda} Q_{\lambda}^{2}, \\
& \sum_{m}^{m}\left(S_{m}^{z}\right)^{2}=1 / 2 \sum_{\lambda} P_{\lambda}^{2}, \\
& \sum_{n}\left(S_{n}^{y}\right)^{2}=1 / 2 \sum_{\lambda} R_{\lambda}^{2},
\end{aligned}
$$

and

$$
\sum_{n}\left(S_{n}^{y}\right)^{2}=1 / 2 \sum_{\lambda} S_{\lambda}^{2}
$$

The Hamiltonian (34) can now be written in terms of the spin waves operators and we have

$$
\begin{aligned}
H=-h_{x} N S_{c}+( & \left.\frac{j}{2}+\frac{h_{x}}{4 S_{c}}\right) \sum_{\lambda}\left(P_{\lambda}^{2}+S_{\lambda}^{2}\right)-2 \sum_{\lambda} P_{\lambda} S_{\lambda} \cos \lambda \\
& +\frac{h_{x}}{4 S_{c}} \sum_{\lambda}\left(Q_{\lambda}^{2}+R_{\lambda}^{2}\right) .
\end{aligned}
$$

A canonical transformation which brings $H$ to normal form is

$$
\begin{aligned}
& P_{\lambda}=\left(-p_{1 \lambda}+p_{2 \lambda}\right) / \sqrt{2}, \\
& S_{\lambda}=\left(p_{1 \lambda}+p_{2 \lambda}\right) / \sqrt{2}, \\
& Q_{\lambda}=q_{1 \lambda} / \sqrt{2}, \\
& R_{\lambda}=q_{2 \lambda} / \sqrt{2} .
\end{aligned}
$$


The Hamiltonian (39) in normal coordinates is then

$$
\begin{aligned}
H=-h_{x} N S_{c} & +\sum_{\lambda}\left\{\left(\frac{j}{2}+\frac{h_{x}}{4 S_{c}}+\cos \lambda\right) p_{1 \lambda}^{2}+\frac{h_{x}}{8 S_{c}} q_{1 \lambda}^{2}\right\} \\
& +\sum_{\lambda}\left\{\left(\frac{j}{2}+\frac{h_{x}}{4 S_{c}}-\cos \lambda\right) p_{2 \lambda}^{2}+\frac{h_{x}}{8 S_{c}} q_{2 \lambda}^{2}\right\} .
\end{aligned}
$$

Writing $H$ as a sum of harmonic oscillators

$$
H=-h_{x} N S_{c}+\sum_{\lambda}\left(\frac{p_{1 \lambda}^{2}}{m_{1 \lambda}}+m_{1 \lambda} \omega_{1 \lambda}^{2} q_{1 \lambda}^{2}\right)+\sum_{\lambda}\left(\frac{p_{2 \lambda}^{2}}{m_{2 \lambda}}+m_{2 \lambda} \omega_{2 \lambda}^{2} q_{2 \lambda}^{2}\right),
$$

with

$$
\begin{array}{ll}
\frac{1}{m_{1 \lambda}}=\left(\frac{j}{2}+\frac{h_{x}}{4 S_{c}}+\cos \lambda\right), & m_{1 \lambda} \omega_{1 \lambda}^{2}=\frac{h_{x}}{8 S_{c}}, \\
\frac{1}{m_{2 \lambda}}=\left(\frac{j}{2}+\frac{h_{x}}{4 S_{c}}-\cos \lambda\right), & m_{2 \lambda} \omega_{2 \lambda}^{2}=\frac{h_{x}}{8 S_{c}} .
\end{array}
$$

and with $q$ and $p$ satisfying the commutation relations

$$
\left[q_{1 \lambda}, p_{1 \lambda}\right]=i=\left[q_{2 \lambda}, p_{2 \lambda}\right]
$$

we can write down the eigenvalues $E$ of $H$

$$
E=-h_{x} N S_{c}+\sum_{\lambda}\left(2 n_{1 \lambda}+1\right) \omega_{1 \lambda}+\sum_{\lambda}\left(2 n_{2 \lambda}+1\right) \omega_{2 \lambda},
$$

where the frequencies of the spin waves are given by

$$
\omega_{1 \lambda}^{2}=\frac{h_{x}}{8 S_{c}}\left(\frac{j}{2}+\frac{h_{x}}{4 S_{c}}+\cos \lambda\right) \quad \omega_{2 \lambda}^{2}=\frac{h_{x}}{8 S_{c}}\left(\frac{j}{2}+\frac{h_{x}}{4 S_{c}}-\cos \lambda\right) .
$$

Since the frequencies $\omega_{1 \lambda}$ and $\omega_{2 \lambda}$ cannot be negative, $h_{x}$ and $j$ must fulfil the inequalities

$$
\frac{j}{2}+\frac{h_{x}}{4 S_{c}}>\cos \lambda \text { and } \frac{j}{2}+\frac{h_{x}}{4 S_{c}}>-\cos \lambda
$$

That is

$$
-\frac{j}{2}-\frac{h_{x}}{4 S_{c}}<\cos \lambda<\frac{j}{2}+\frac{h_{x}}{4 S_{c}} \text { for all } \lambda .
$$

One way to ensure that this is always the case is to require that

$$
\frac{j}{2}+\frac{h_{x}}{4 S_{c}}=\frac{j}{2}+\frac{h_{x} \sqrt{3}}{6} \geq 1
$$

The unfortunate implication of equation (48) is that our spin waves theory will be valid only for large values of the transverse field $h_{x}$ and that we will be kept in the dark concerning the characteristics of the ANNNI model in the presence of a weak transverse external magnetic field. In the ground state, all $n_{\lambda}=0$ and we have for the ground state energy

$$
E_{g}=-h_{x} N S_{c}+\sum_{\lambda} \sqrt{\frac{h_{x}}{8 S_{c}}\left(\frac{j}{2}+\frac{h_{x}}{4 S_{c}}+\cos \lambda\right)}+\sum_{\lambda} \sqrt{\frac{h_{x}}{8 S_{c}}\left(\frac{j}{2}+\frac{h_{x}}{4 S_{c}}-\cos \lambda\right)} .
$$

Here, as in [1], the frequencies of the spin waves fall into two categories. However, unlike in the Heisenberg model studied by Anderson, $\omega_{1 \lambda}$ and $\omega_{2 \lambda}$ are not identical and furthermore the dispersion laws are quite different for long wavelengths. In fact, for $\lambda \rightarrow 0$, the oscillator frequencies are quadratic in $\lambda$ as 


$$
\omega_{1 \lambda} \approx \alpha+\beta \lambda^{2},
$$

where

$$
\begin{aligned}
& \alpha=\left(\frac{h_{x}}{4 S_{c}}\right)^{1 / 2}\left[\left(\frac{j}{2}+\frac{h_{x}}{4 S_{c}}+1\right)^{1 / 2}+\left(\frac{j}{2}+\frac{h_{x}}{4 S_{c}}-1\right)^{1 / 2}\right] \\
& \text { and } \beta=\frac{1}{4}\left(\frac{h_{x}}{4 S_{c}}\right)^{1 / 2}\left[\left(\frac{j}{2}+\frac{h_{x}}{4 S_{c}}-1\right)^{-1 / 2}-\left(\frac{j}{2}+\frac{h_{x}}{4 S_{c}}+1\right)^{-1 / 2}\right]
\end{aligned}
$$

This quadratic dispersion law is rather characteristic of the ground state of the ferromagnetic Heisenberg model [3]. Anderson on the other hand predicted a linear dispersion law for the Heisenberg antiferromagnet [5].

\section{Spin waves theory ground state energy of the spin $-1 / 2$ ANNNI model in a transverse field}

We are now in a position to calculate the ground state energy for an infinite chain, but first let us calculate the average values of the kinetic and potential energy terms that occur in $H$ since we will need them later for calculating order parameters.

If we write

$$
H_{1}=\left(\frac{j}{2}+\frac{h_{x}}{4 S_{c}}+\cos (\lambda)\right) p_{1 \lambda}^{2}+\frac{h_{x}}{8 S_{c}} q_{1 \lambda}^{2}
$$

with energy $H_{1 \lambda}=\left(2 n_{1 \lambda}+1\right) \omega_{1 \lambda}$

and

$$
H_{2}=\left(\frac{j}{2}+\frac{h_{x}}{4 S_{c}}+\cos (\lambda)\right) p_{2 \lambda}^{2}+\frac{h_{x}}{8 S_{c}} q_{2 \lambda}^{2}
$$

with energy $\mathrm{H}_{2 \lambda}=\left(2 \mathrm{n}_{2 \lambda}+1\right) \omega_{2 \lambda}$, then in the ground state

$$
<H_{1}>=E_{1 g}=\left(\frac{j}{2}+\frac{h_{x}}{4 S_{c}}+\cos (\lambda)\right)<p_{1 \lambda}^{2}>+\frac{h_{x}}{8 S_{c}}<q_{1 \lambda}^{2}>=\omega_{1 \lambda},
$$

and since

$$
\left\langle q_{1 \lambda}^{2}\right\rangle=\left\langle p_{1 \lambda}^{2}\right\rangle,
$$

we have

$$
\begin{aligned}
& <p_{1 \lambda}^{2}>=\frac{\omega_{1 \lambda}}{2\left(\frac{j}{2}+\frac{h_{x}}{4 S_{c}}+\cos (\lambda)\right)} \\
& =\frac{h_{x}}{16 \omega_{1 \lambda} S_{c}}
\end{aligned}
$$

and 
$<q_{1 \lambda}^{2}>=\frac{4 \omega_{1 \lambda} S_{c}}{h_{x}}$

That is, substituting the frequencies (45)

$$
<p_{1 \lambda}^{2}>=\frac{1}{2} \sqrt{\frac{h_{x} / 8 S_{c}}{\left(\frac{j}{2}+\frac{h_{x}}{4 S_{c}}+\cos \lambda\right)}}
$$

and

$$
<q_{1 \lambda}^{2}>=\frac{1}{2} \sqrt{\frac{\left(\frac{j}{2}+\frac{h_{x}}{4 S_{c}}+\cos \lambda\right)}{h_{x} / 8 S_{c}}}
$$

in the ground state. Similarly,

$$
<p_{2 \lambda}^{2}>=\frac{1}{2} \sqrt{\frac{h_{x} / 8 S_{c}}{\left(\frac{j}{2}+\frac{h_{x}}{4 S_{c}}-\cos \lambda\right)}}
$$

and

$$
<q_{2 \lambda}^{2}>=\frac{1}{2} \sqrt{\frac{\left(\frac{j}{2}+\frac{h_{x}}{4 S_{c}}-\cos \lambda\right)}{h_{x} / 8 S_{c}}}
$$

in the ground state.

In order to compute the spin waves theory ground state energy of the spin-1/2 ANNNI model in a transverse field, we replace the sum over $\lambda$ in equation (49) by an integral and write

$E_{g}=-h_{x} N S_{c}+\frac{N}{2}\left(\frac{h_{x}}{8 S_{c}}\right)^{1 / 2} \frac{1}{2 \pi} \int_{-\pi}^{\pi}\left\{\left(\frac{j}{2}+\frac{h_{x}}{4 S_{c}}+\cos \lambda\right)^{1 / 2}+\left(\frac{j}{2}+\frac{h_{x}}{4 S_{c}}-\cos \lambda\right)^{1 / 2}\right\} \mathrm{d} \lambda$

The factor of $N / 2$ comes from the fact that there are $N / 2$ values of the wave number $\lambda$.

Evaluating the above integral, we obtain

$E_{g}=-h_{x} N S_{c}+\frac{N}{\pi}\left(\frac{h_{x}}{8 S_{c}}\right)^{1 / 2}\left\{(\gamma-1)^{1 / 2} \mathrm{E}\left(i \sqrt{2}(\gamma-1)^{-1 / 2}\right)+(\gamma+1)^{1 / 2} \mathrm{E}\left(\sqrt{2}(\gamma+1)^{-1 / 2}\right)\right\}$,

where $E$ is an elliptic integral of the second kind and

$$
\gamma=\frac{j}{2}+\frac{h_{x}}{4 S_{c}}
$$

We note that $\mathrm{E}\left(i \sqrt{2}(\gamma-1)^{-1 / 2}\right)$ is a real function of $\gamma$. 


\section{CONCLUSION}

We have successfully applied the spin wave theory to classically determine the ground state energy of the ANNNI model and the ANNNI model in a strong transverse field. Long range order was predicted for the one dimensional antiferromagnetic ANNNI model, as opposed for example to the situation with the Heisenberg model for which there is no long range order.

\section{Acknowledgments}

The author is grateful to the DAAD (German Academic Exchange Service) for a scholarship and thanks the University of Bayreuth, Germany, for hospitality.

\section{References:}

[1] Bloch, F., Zur Theorie des Ferromagnetismus, Z. Physik 61 (1930) 3-4, 206-219. DOI: 10.1007/BF01339661

[2] Heller, G., Kramers, H.A., Ein klassisches Modell das Ferromagnetikums und seine nacträgliche Quantiserung im Gebiete tiefer Temepraturen, Proceedings Koninklijke Akademie van Wetenschappen 37 (1934) 378-385.

[3] HulthÉN, L., Über das antiferromagnetische Austauschproblem bei tiefen Temperaturen. Proc. K. Akad. Wet. Amsterdam 39 (1936) 2, 190-200.

[4] Bethe, H.A., Zur Theorie der Metalle, Z. Physik 71 (1931) 3-4, 205-226.

[5] Anderson, P.W., An Approximate Quantum Theory of the Antiferromagnetic Ground State, Phys. Rev. 86 (1952) 5, 694-701. DOI: http://dx.doi.org/10.1103/PhysRev.86.694

[6] GAIDIDEI, Y., BuetTNER, H., Effects of spin-elastic interactions in frustrated Heisenberg antiferromagnets, Phys. Rev. B 62, (2000) 8604-8607. 